\title{
Considerations for point-of-care diagnostics: evaluation of acridine orange staining and postprocessing methods for a three-part leukocyte differential test
}

\author{
Amy J. Powless \\ Roxanna J. Conley \\ Karan A. Freeman \\ Timothy J. Muldoon
}




\title{
Considerations for point-of-care diagnostics: evaluation of acridine orange staining and postprocessing methods for a three-part leukocyte differential test
}

\author{
Amy J. Powless, ${ }^{a}$ Roxanna J. Conley, ${ }^{b}$ Karan A. Freeman, ${ }^{b}$ and Timothy J. Muldoon ${ }^{a, *}$ \\ aUniversity of Arkansas, Department of Biomedical Engineering, Fayetteville, Arkansas, United States \\ bUniversity of Arkansas, Pat Walker Health Center, Fayetteville, Arkansas, United States
}

\begin{abstract}
There exists a broad range of techniques that can be used to classify and count white blood cells in a point-of-care (POC) three-part leukocyte differential test. Improvements in lenses, light sources, and cameras for image-based POC systems have renewed interest in acridine orange $(\mathrm{AO})$ as a contrast agent, whereby subpopulations of leukocytes can be differentiated by colorimetric analysis of AO fluorescence emission. We evaluated the effect on test accuracy using different $\mathrm{AO}$ staining and postprocessing methods in the context of an image-based POC colorimetric cell classification scheme. Thirty blood specimens were measured for percent cell counts using our POC system and a conventional hematology analyzer for comparison. Controlling the $\mathrm{AO}$ concentration used during whole-blood staining, the incubation time with $\mathrm{AO}$, and the colorimetric ratios among the three population of leukocytes yielded a percent deviation of $0.706 \%,-1.534 \%$, and $-0.645 \%$ for the lymphocytes, monocytes, and granulocytes, respectively. Overall, we demonstrated that a redshift in AO fluorescence was observed at elevated $A O$ concentrations, which lead to reproducible inaccuracy of cell counts. This study demonstrates there is a need for a strict control of the AO staining and postprocessing methods to improve test accuracy in these POC systems. (C) The Authors. Published by SPIE under a Creative Commons Attribution 3.0 Unported License. Distribution or reproduction of this work in whole or in part requires full attribution of the original publication, including its DOI. [DOI: $10.1117 / 1$. JBO.22.3.035001]
\end{abstract}

Keywords: acridine orange; three-part leukocyte differential; point-of-care; leukocyte staining method; white blood cells; blood test accuracy.

Paper 160830R received Dec. 1, 2016; accepted for publication Feb. 8, 2017; published online Mar. 2, 2017.

\section{Introduction}

Point-of-care (POC) blood analyzers have the potential to provide rapid, portable blood tests in rural and low resource settings or at a patient's bedside when cost, reagents, infrastructures, and available technicians prevent these tests from being accessible. ${ }^{1,2}$ For example, a three-part leukocyte differential test, which yields a percentage count among the three main cell populations (granulocytes, lymphocytes, and monocytes), could indicate a myriad of abnormalities such as sepsis, malignancy, or infection based on perturbations of the count. ${ }^{3,4} \mathrm{~A}$ five-part differential test is also available and would provide more information including a count of the basophils and eosinophils; however, a three-part differential is typically sufficient for most tests with the assumption that basophil and eosinophil levels are normal and, thus, are not required to be reported. ${ }^{3}$ Currently, a three-part differential test is performed on clinical hematology analyzers, common in clinics and central laboratories in the United States, that utilize flow cytometry techniques (Coulter principle based on electrical impedance, fluorescence, or both) that involve single cell analysis. ${ }^{5}$ These systems are not appropriate for POC approaches due to the per-test cost ( $\$ 0.25$ to $\$ 1.25$ for reagents alone), cell counts $(10,000$ to

*Address all correspondence to: Timothy J. Muldoon, E-mail: tmuldoon@uark .edu
50,000 cells/s) that require many reagents, bulky and heavy equipment, and overall cost $(\sim \$ 10,000$ to $\$ 25,000))^{2,6}$

POC devices are typically inexpensive (ex. $<\$ 10$ in mass production, estimated prototype cost of \$240) and relatively light weight for portability (ex. $12 \times 9 \times 5$ in. dimensions, a cellphone adaptor weighing $\sim 18 \mathrm{~g}$ ) and have the advantage of providing test results at or near a patient. ${ }^{7-13}$ In the United States, a Clinical Laboratory Improvement Amendments (CLIA)-waived test classification is required to use these devices in a clinical setting. It defines the test accuracy of a leukocyte differential to be within $\pm 15 \%$ of a clinical hematology analyzer while being simple enough for an inexperienced user to operate. ${ }^{14-16}$ On a global health scale, the requirements for this test classification have been used as a reference in designing POC devices. ${ }^{8,17}$ A common challenge with achieving this accuracy is in the monocyte count since it represents the smallest population of leukocytes ( $4 \%$ to $10 \%$ ). ${ }^{18,19}$ For instance, an arbitrary total count of 10,000 cells would only consist of 400 to 1000 monocytes while only 4 to 10 monocytes would be available to count in a population of 100 cells, which reduces the likelihood of detecting that small population. ${ }^{19}$ While many POC blood analyzers have achieved results within CLIA standards, ${ }^{9,12,20}$ the combination of the device simplicity and accurate monocyte counts can be challenging design factors.

Current POC blood analyzers aim to significantly reduce cost below that of conventional hematology analyzers $(>\$ 20,000)$, 
as well as operational complexity by removing the need for highly trained technicians. ${ }^{7,8}$ In recent years, a small impedance-based bench-top system that reduces overall system cost and size has been approved for clinical use as a POC device. ${ }^{1}$ For this device and other similar devices, miniaturizing the system using the combination of an impedance technique (Coulter principle) and microfluidics (microchannels) is a common approach to reduce cost and complexity by simply minimizing optical components and reagents. ${ }^{10,13,21,22}$ Some disadvantages to impedance-based measurements include electrolysis, gas formation, and the need for red blood cell (RBC) lysis and dilution reagents, all of which add design complexity and, potentially, operational cost. ${ }^{10,23-25}$

Alternatively, there are many different types of image-based methods that have become popular due to their reduced size and cost from the development of low-cost plastic optics, light-emitting diodes (LEDs), and other new optical technologies. There are label-free techniques that require no contrast agents but rely on intrinsic properties where some are based on projections of the cells, such as lens-free shadow imaging, white light diffraction phase microscopy/quantitative phase imaging, and lensless incoherent holography. ${ }^{26-28}$ Another approach uses fluorescence imaging since the optical system can be simplified and easily incorporated into low-cost imaging platforms such as cell phones and microcomputers, for example, 3-D printed compact devices attached to cell phones, plastic lenses developed via diamond turning, and small microfluidic-based blood cassettes that automatically combine the blood with the fluorescent dye without the need of additional reagents. ${ }^{11,12,20}$ Overall, these systems have reported adequate results; however, further work needs to be done to optimize for POC leukocyte counting.

Emerging optical technologies for fluorescence microscopy enable the use of low-cost lenses, filters, LEDs, and detectors, such as a cell-phone-integrated camera, in which the spatial resolution, quantum efficiency, and frame rates have improved to where high-quality imaging is practical. ${ }^{11,17}$ This has renewed the interest in the use of acridine orange (AO), a fluorescent vital dye that has been used in medical diagnostics since the 1950s for uses in diagnosing and detecting gynecological cancer, microorganisms in cerebrospinal fluid, and malaria. ${ }^{29-32}$ AO only stains nucleated cells and, therefore, does not require additional reagents (ex. RBC lysis and dilution reagents), in contrast to other POC systems. This is due to the amphipathic structure of $\mathrm{AO}$ that enables the molecules to pass through the cell and nuclear membranes and intercalate the major and minor grooves of DNA, leading to a strong nuclear to cytoplasmic contrast in living cells. ${ }^{33,34}$ The most promising advantage of staining whole blood with $\mathrm{AO}$ are the colorimetric features that can be extracted from leukocytes that are distinct to each cell population. AO $\left(\lambda_{\mathrm{ex}}=455 \mathrm{~nm}\right)$ will yield a range of fluorescence emission (from 525 to $650 \mathrm{~nm}$ ), contingent on intracellular environmental factors such as local $\mathrm{pH} .{ }^{33,35}$ These local variations in intracellular $\mathrm{pH}$ are dependent on the presence of intracellular lysosomes, vesicles, and other components specific to the role that each leukocyte population plays; this enables differentiation and classification of each cell population based on its colorimetric features following AO staining. ${ }^{33,36}$ In Fig. 1, granulocytes represent the most acidic cell population containing many acidic vesicles resulting in the greatest red emission. ${ }^{35,36}$ The lymphocytes have minimal cytoplasm with the least acidic cellular content resulting in the greatest green emission. Monocytes have more cytoplasm but less acidic content and, thus, are between

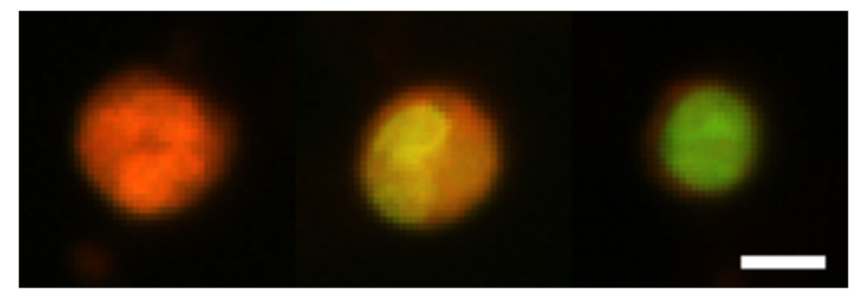

(a)

(b)

(c)

Fig. 1 Example images of AO-stained leukocytes: (a) granulocyte, (b) monocyte, and (c) lymphocyte. Scale bar $=10 \mu \mathrm{m}$. (Images enhanced for publication.)

the emission spectrum of the other cell populations. Many POC devices utilize this colorimetric feature ${ }^{21,35}$ by comparing each cell's red-to-green fluorescence intensity ratio ( $R G$ ratio). ${ }^{20,37,38}$ In postprocessing, a histogram plot of the frequency of RG ratios can reveal three peaks indicative of each cell population. The challenge in using this method is in the monocyte count because the peak can be too small to distinguish, creating issues in defining the dividing lines (cutoff values) between peaks. For instance, using a static approach where the cutoff values are constant for all tests, the peaks could shift slightly but enough to miscount the cells.

The accuracy of a leukocyte differential using AO as a contrast agent can be limited by its variability in results based on changes in the staining method. There are many published methods with varied incubation periods between 5 and $10 \mathrm{~min}$ and final concentrations between 10 and $50 \mu \mathrm{g} / \mathrm{ml} .^{17,33,37}$ It has been demonstrated that over time AO produces an overall redshift due to accumulation of the dye in the cytoplasm and acidic vacuoles, which could affect the differential result accuracy. ${ }^{33,36}$ This restricts the amount of cells that can be counted in a given time based on RG ratios, thus reducing the probability of detecting all of the small population of monocytes.

Since there are a variety of AO staining protocols for whole blood, there is a need for an optimized method to account for the effect of incubation period and concentration, relating to a rapid redshift in the $\mathrm{AO}$ fluorescence emissions, on the differential test results. In this paper, we investigated three variables involved in the staining and postprocessing method: AO staining concentration, incubation period, and RG colorimetric parameters. First, 10 AO staining concentrations and three incubation periods were evaluated together with a preassigned RG colorimetric parameter, and the optimal method was based on the lowest deviation from the clinical results. Next, using the optimized staining method, the RG colorimetric parameters were varied and evaluated based on the deviation between the two systems. Then, the combination of those optimal factors was used to compare three time gates that were related to the incubation period. All the experiments involved the comparison of all three subpopulations individually between our system and the hematology analyzer. The final results were analyzed for agreement and correlation to the clinical values to determine overall test accuracy.

\section{Materials and Methods}

\subsection{System Overview and Image Acquisition}

The imaging system used in these experiments was designed as a POC fluorescence-based system with low-cost components, such as the microcomputer and LED excitation source, as shown in Fig. 2. This system will be referred to as the POC system in 


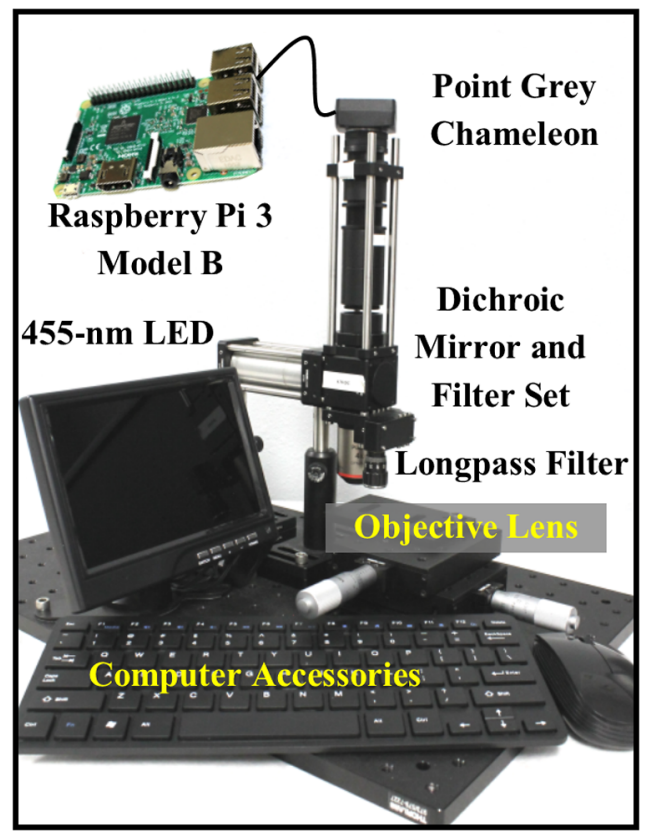

Fig. 2 The imaging system designed to mimic a POC device. It consists of a Raspberry Pi microcomputer connected to an epi-fluorescence microscope with a $4 \times$ Nikon Plan Fluor objective, a 500-nm long pass filter, a 475-nm dichroic mirror and filter set, a 455-nm LED, and a Point Grey Chameleon color camera.

this paper. The Raspberry Pi 3 Model B microcomputer $(\$ 40$, Adafruit) was connected to an epi-fluorescence microscope consisting of a 455-nm LED illumination (Philips), a $4 \times$ Nikon Plan Fluor objective (NA $=0.13$, Nikon Instruments Inc.), a dichroic mirror (475-nm cutoff wavelength, Chroma Technologies), a 500-nm longpass emission filter (Thorlabs), a achromatic doublet (100-mm focal length, Thorlabs), and a USB 2.0 Chameleon color camera (CMLN-13S2C-CS, Point Grey). All images were acquired with an optical magnification of $\sim 5.81 \times$, a field of view (FOV) of $1.89 \times 1.42 \mathrm{~mm}$, an exposure time of $150 \mathrm{~ms}$, and a gain of $0 \mathrm{~dB}$. For each image set, six nonoverlapping FOVs were acquired within 1 min intervals.

\subsection{Blood and Acridine Orange Preparation}

To study the effect of AO staining concentration, we performed a human subject trial in cooperation with the University of Arkansas Pat Walker Health Center. Following informed consent, blood samples were collected from 30 healthy subjects under the approval of the University of Arkansas Institutional Review Board (IRB 15-11-384). The samples were drawn via venipuncture (4-ml whole blood) and were analyzed at least 20 min later on a clinical hematology analyzer (XS 1000i, Sysmex) after gently inverting 10 times. An AO stock solution was prepared by mixing distilled water with AO hemi(zinc chloride) salt (Sigma-Aldrich) for a concentration of $100 \mu \mathrm{g} / \mathrm{ml}$; it was maintained at a $\mathrm{pH}$ of 7.4 , stored at $4^{\circ} \mathrm{C}$, and protected from light. This stock solution was diluted into nine concentrations ranging from 10 to $90 \mu \mathrm{g} / \mathrm{ml}$. Whole blood was stained at a 1:1 ratio with the 10 concentrations including the stock concentration producing final concentrations ranging from 5 to $50 \mu \mathrm{g} / \mathrm{ml}$. Image acquisition using the POC System was performed within $4 \mathrm{~h}$ of the blood draw at room temperature or $24 \mathrm{~h}$ if the samples were refrigerated.

\subsection{Automated Differential Algorithm}

We have developed an image analysis-based automated differential algorithm, shown in Fig. 3, to calculate the three-part leukocyte differential based on the red to green fluorescence intensity ratio (RG ratio). Following image acquisition, the individual cells were segmented by applying a global threshold to convert the image to a binary image that was applied as a mask. Any object with an area less than 15 pixels and greater than 100 pixels were considered platelets, clusters of cells, and debris, and were therefore excluded. The RG ratio was calculated for each cell by dividing the mean red intensity by the mean green intensity. These intensity values were acquired from the red and green grayscale images separated from the RGB images. Those values were plotted on a histogram displaying the frequency of cells at a certain $\mathrm{RG}$ ratio value. The three groups of peaks in the histogram [Fig. 3(d)] indicating the three cell populations were used to define cutoff ranges consisting of the lymphocyte/monocyte (LM) and monocyte/granulocyte (MG) cutoffs. We chose a static approach to define fixed cutoff ranges to avoid issues with variations in the peaks [Fig. 3(c)] among samples. The initial cutoff values ( 0.42 and 0.59$)$ were based on visual inspection of the groups of peaks from the average of the histogram in Fig. 3(c) and four other histograms. From those ranges, the three-part differential was calculated by automatically counting the number of cells within each cutoff section.

\subsection{Investigation of Three Variables in the Overall Staining and Postprocessing Method}

Three variables within the overall staining and postprocessing method were analyzed to determine their effect on the differential results (Fig. 4). This included concentration (variable 1) and time gates representing a sample of incubation periods (variable 2) within the staining method and post hoc analysis of the RG ratio cutoff values (variable 3 ).

To evaluate the staining method, $10 \mathrm{AO}$ concentrations varying from 5 to $50 \mu \mathrm{g} / \mathrm{ml}$ (final) and incubation periods separated into three time gates ( 3 to 4,5 to 6 , and 7 to $8 \mathrm{~min}$ ) were used. The $10 \mathrm{AO}$ concentrations were selected based on other existing AO staining methods. ${ }^{17,33,37}$ The time gates were determined based on the need to acquire a large number of cells while minimizing the time-dependent redshift in AO emission. The optimal AO concentration and time gate were analyzed simultaneously with percent deviation from the clinical hematology analyzer as a measure of accuracy. The images were acquired using the three time gates for each of the 10 concentrations, resulting in 30 different image sets per specimen.

For post hoc analysis of the postprocessing method, the RG ratio cutoffs between the LM and MG were varied. Image data acquired using time gate 5 to $6 \mathrm{~min}$ and $10 \mu \mathrm{g} / \mathrm{ml}$ from all samples were evaluated with the different cutoff values. Initially, the cutoff range was manually selected via visual inspection of histograms with very distinct peaks separating the three populations. However, since this process could introduce observer bias, a range of cutoff values near these were evaluated. Five values from each cutoff (LM $0.42 \pm 0.2$, MG $0.59 \pm 0.2$, in increments of 0.1 ) were applied to those image sets comprising 25 different results per specimen. The results were analyzed for percent deviation from the clinical hematology analyzer as a measure of accuracy. 


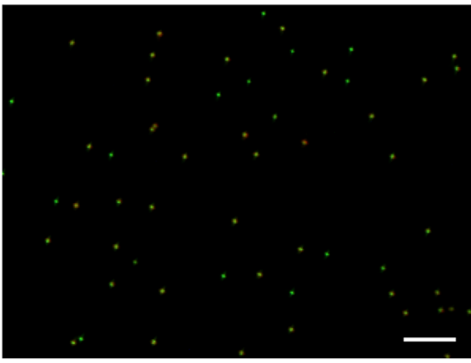

(a) Select individual cells Exclude objects less than 15 pixels (platelets) and greater than 100 pixels (clusters and debris)

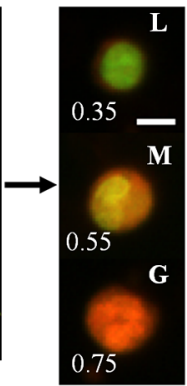

(b) Calculate RG ratio (c)

Plot the frequency of RG ratios on a histogram Exclude RG ratios $<1.2$ (platelets and debris)

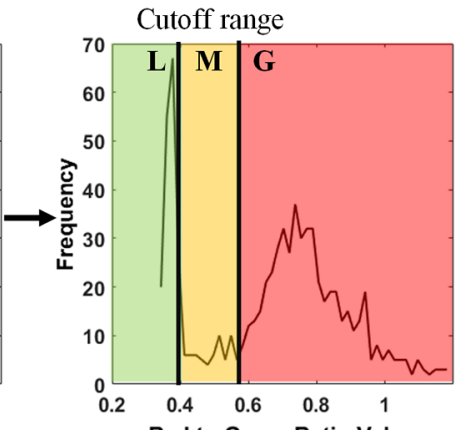

(d)

Define classification cutoff ranges for each cell type

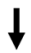

Three-part differential (normal range) ${ }^{18}$

Red-green ratio $=\frac{\text { mean red intensity }}{\text { mean green intensity }}$
Lymphocytes $=20 \%$ to $45 \%$

Monocytes $=4 \%$ to $10 \%$

Granulocytes $=40 \%$ to $76 \%$

Fig. 3 An overview of the image analysis-based automated differential algorithm. (a) Each cell per image was segmented and measured for mean red and green fluorescence intensity to (b) calculate the red-togreen (RG) ratio. (c) The $R G$ ratios were plotted on a histogram showing the frequency of each ratio value. (d) $R G$ ratio cutoff range (bold lines) was used to calculate the final three-part leukocyte differential results.

Variable 1: Concentration

\begin{tabular}{|l|l|l|l|l|l|l|l|l|l|}
\hline \multicolumn{10}{|c|}{ Final concentration $(\boldsymbol{\mu g} / \mathbf{m l})$} \\
\hline 5 & 10 & 15 & 20 & 25 & 30 & 35 & 40 & 45 & 50 \\
\hline \multicolumn{10}{|c}{}
\end{tabular}

Variable 2: Time gates

\begin{tabular}{|c|c|c|c|c|c|c|}
\hline $\begin{array}{c}\begin{array}{c}\text { Staining of } \\
\text { Whole Blood }\end{array} \\
\text { Whole blood mixed } \\
\text { with AO solution (1:1) }\end{array}$ & $\mathrm{min}$. & & & & & \\
\hline Vari & ble 3: & R r & 0 cut & $s(\mathrm{Pc}$ & hoc) & \\
\hline & & ipho & $\mathrm{e} / \mathbf{M}$ & cyte & toff & ues \\
\hline & & 0.40 & 0.41 & 0.42 & 0.43 & 0.44 \\
\hline & 0.57 & 1 & 2 & 3 & 4 & 5 \\
\hline Monocyte/ & 0.58 & 6 & 7 & 8 & 9 & 10 \\
\hline Granulocyte & 0.59 & 11 & 12 & 13 & 14 & 15 \\
\hline & 0.60 & 16 & 17 & 18 & 19 & 20 \\
\hline & 0.61 & 21 & 22 & 23 & 24 & 25 \\
\hline
\end{tabular}

Fig. 4 The analysis method consisting of three variables: (1) concentration, (2) time gates, and (3) RG ratio cutoffs. During data collection, images were acquired at 10 different concentrations for each time gate. In post hoc analysis, a fixed set of time gate and concentration data was used to determine optimal $R G$ ratio cutoff values when the range was varied.

\subsection{Analysis of the Combined Optimal Staining and Postprocessing Methods}

All 30 samples measured in triplicates (for repeatability of the system) were used to test the accuracy of the combined optimal staining and postprocessing methods based on the results from the previous section. Three time gates from 3 to 4,5 to 6 , and 7 to $8 \mathrm{~min}$ were used to demonstrate the redshift that occurs over time and how it affects the test results. Two metrics were used in the analysis: Bland-Altman and correlation. For the BlandAltman analysis, the mean difference between the results produced by the POC system and the clinical hematology analyzer 
was used to calculate the average bias between the systems. To determine the correlation between the systems, all values were plotted and a linear regression was drawn to produce an $R$-squared value with a value closer to one indicative of a closer relation to the clinical value. All three time gates were compared using this same analysis approach.

\section{Results}

\subsection{Acridine Orange Concentration on Cell Count Accuracy}

AO concentration effects on overall leukocyte differential accuracy are summarized in Fig. 5. The green, blue, and red bars represent the deviation from the clinical standard measurement for each cell population (lymphocytes, monocytes, and granulocytes, respectively). The time gates were included since it is a dependent variable in the $\mathrm{AO}$ staining method. The monocyte population demonstrated the greatest inaccuracy at concentrations above $10 \mu \mathrm{g} / \mathrm{ml}$ where the deviations exceeded the CLIAdefined limits of $\pm 15 \%$, and many exceeded $100 \%$. Similarly, lymphocytes had the greatest inaccuracy at concentrations above
$15 \mu \mathrm{g} / \mathrm{ml}$; however, the deviations were negative, indicating that the POC system was underestimating the prevalence of this cell population. Overall, a concentration of $10 \mu \mathrm{g} / \mathrm{ml}$ and time gate 5 to 6 min, denoted by asterisks in Fig. 5, demonstrated the lowest deviations (lymphocytes $=-2.47 \%$, monocytes $=9.87 \%$, and granulocytes $=-0.192 \%$ ) from the clinical hematology analyzer.

In Fig. 6, a histogram plot demonstrates the change in the leukocyte frequency peaks when the concentration was increased and the time gate was maintained at 5 to $6 \mathrm{~min}$. In lower concentrations, the peaks were in locations that match the fluorescence characteristics of each cell population, consistent with previous results at $10 \mu \mathrm{g} / \mathrm{ml}$. With increased concentrations, the largest peak shifted to the right (redshifts) and expanded where there was less distinction between the groups.

\subsection{RG Ratio Cutoff Effects on Cell Count Accuracy}

The RG ratio cutoff values used in the differential algorithm to count the cell populations were evaluated after observing the effects from variables 1 (concentration) and 2 (time gates). In Fig. 7, similar to the previous section, green, blue, and red

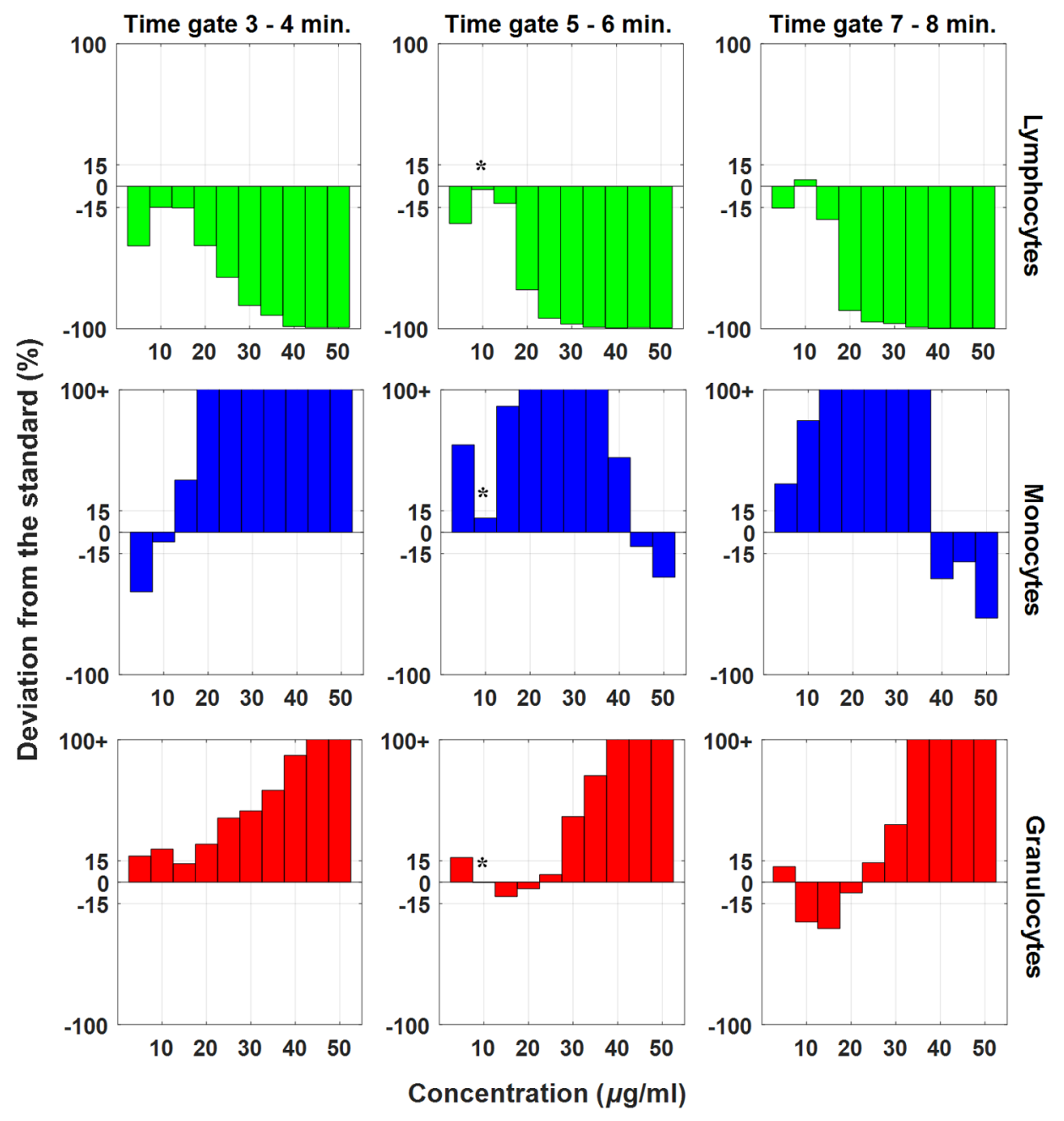

Fig. 5 A comparison of different concentrations (columns) and time gates (row) using percent deviation as a measure of accuracy. The green, blue, and red plots represent the lymphocytes, monocytes, and granulocytes, respectively. A concentration of $10 \mu \mathrm{g} / \mathrm{ml}$ and time gate between 5 and 6 min demonstrated the lowest deviations (lymphocytes $=-2.47 \%$, monocytes $=9.87 \%$, and granulocytes $=$ $-0.192 \%)$, denoted by an asterisk. 


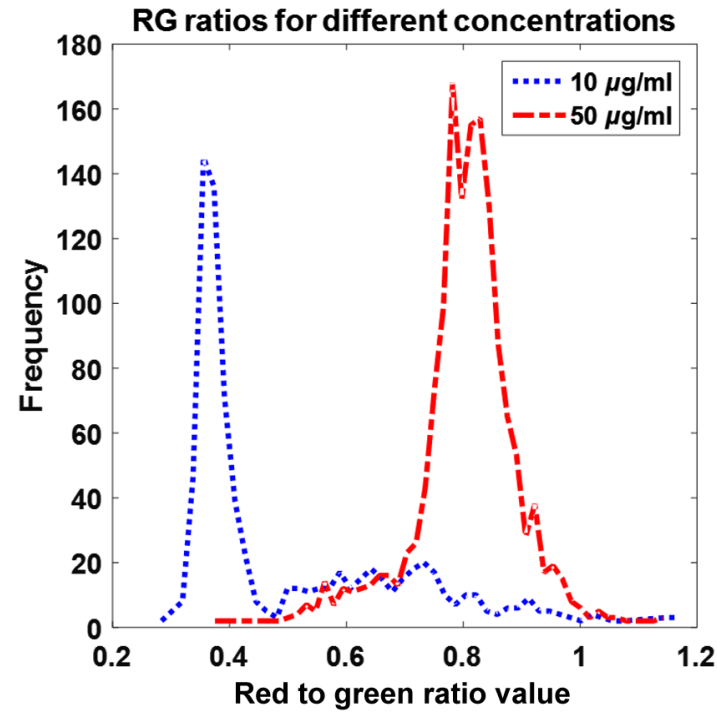

Fig. 6 Histograms demonstrating the change in the peaks when concentrations were increased and the time gate was maintained at 5 to $6 \mathrm{~min}$. The dotted line represents a concentration of $10 \mu \mathrm{g} / \mathrm{ml}$, where there are peaks in expected locations $(n=754$ cells). The dashed line represents the highest concentration of $50 \mu \mathrm{g} / \mathrm{ml}$, where the peaks are less distinct and the first peak with a greater bandwidth ( $n=1457$ cells). bars indicate the three cell populations. For granulocytes and lymphocytes, all cutoff values were between $-3.7 \%$ to $2.05 \%$ and $-11.01 \%$ to $4.14 \%$, respectively. Monocytes, as anticipated, demonstrated the greatest variability with half of the tested RG cutoff values producing deviations greater than $\pm 15 \%$ compared to the clinical results. Additionally, there were observable trends in deviation values for the lymphocytes and granulocytes, relating to the cutoff value (LM or MG) being studied. For lymphocytes, the LM cutoff directly affected the count by accepting more or less cells between the first two peaks on the histogram [Fig. 3(d)]; therefore, there was greater plot-to-plot variation in accuracy. This result was similar for the granulocyte count except that the trend varied within each plot. The cutoff values of 0.42 (LM) and $0.58(\mathrm{MG})$, denoted by asterisks in Fig. 7, produced the lowest deviations from the clinical results (lymphocytes $=0.706 \%$, monocytes $=-1.534 \%$, and granulocytes $=-0.645 \%$ ). This demonstrates the importance of controlling the staining process since slight variations in the $\mathrm{RG}$ ratio cutoff values affected the cell count accuracy.

\subsection{Time Gate Effects on Cell Count Accuracy}

From the previous results in Sec. 3.2, the optimal staining and postprocessing method included a final concentration of $10 \mu \mathrm{g} /$ $\mathrm{ml}$ and the RG ratio cutoff values of $0.42(\mathrm{LM})$ and $0.58(\mathrm{MG})$. Using these constraints, Bland-Altman analysis and linear

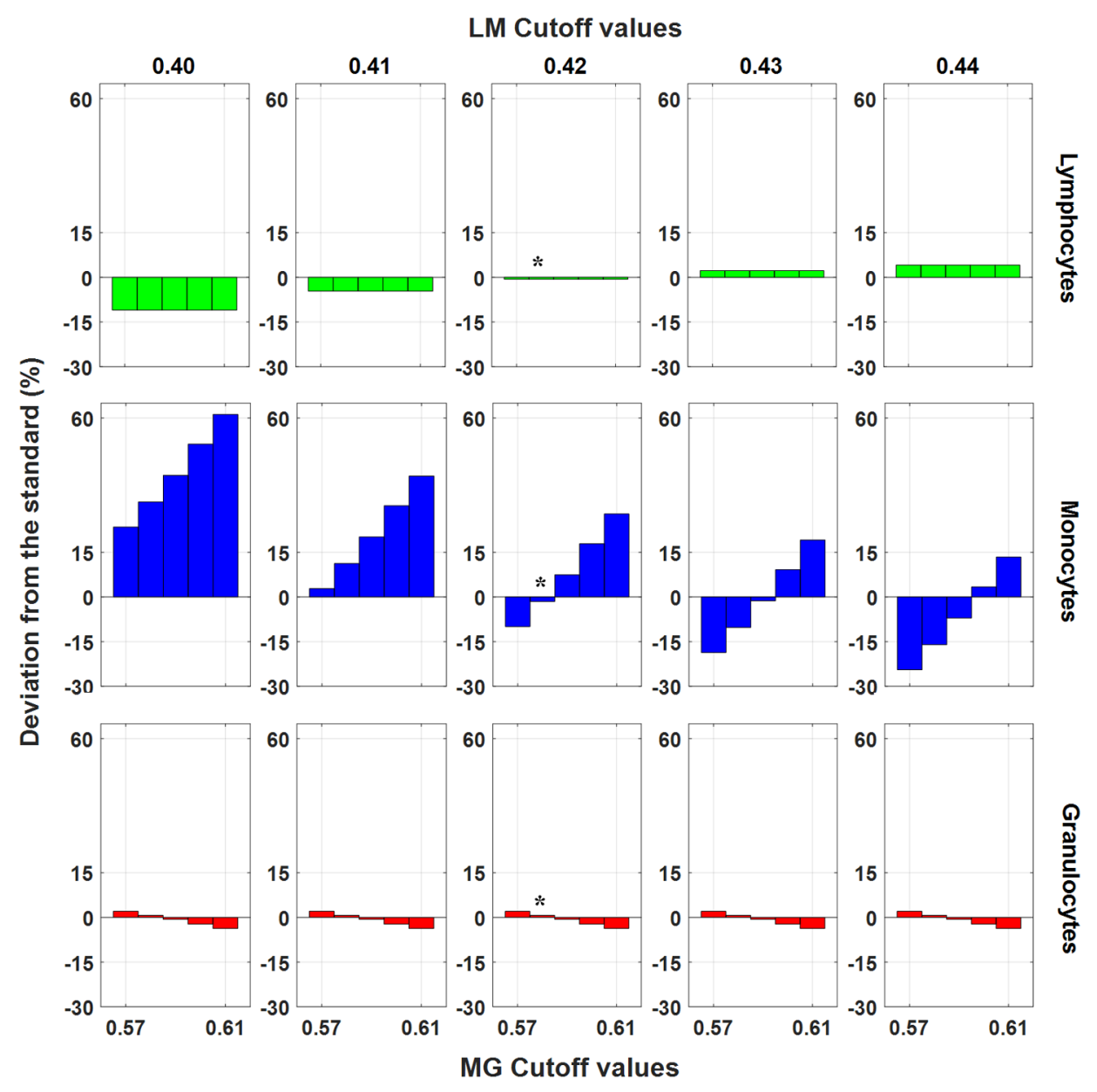

Fig. 7 A comparison of different cutoff values between LM ( 0.40 and 0.44$)$ and MG ( 0.57 and 0.61$)$ using percent deviation as a measure of accuracy using data from the optimal staining method. The cutoff values of $0.42(\mathrm{LM})$ and $0.58(\mathrm{MG})$ produced the lowest deviations from the clinical hematology analyzer (lymphocytes $=0.706 \%$, monocytes $=-1.534 \%$, and granulocytes $=-0.645 \%$ ), denoted by asterisks . 
correlation were used to compare our POC system to the clinical hematology analyzer for the three time gates at 3 to 4,5 to 6 , and 7 to 8 min. In Fig. 8 (left), the Bland-Altman plots demonstrate the agreement between the two systems with the solid lines representing the mean difference (bias) and the dashed lines representing the $95 \%$ confidence intervals of the differences (limits of agreement) calculated by the mean \pm 1.96 times the standard deviation. ${ }^{6,21,26} \mathrm{~A}$ bias at zero represents perfect agreement between the systems, and the closer the limits of agreement are to this bias, the less likely our POC system will produce different results from the clinical hematology analyzer results that could affect the diagnosis. For example, data points above the mean represent an overestimate of that population, which could lead to a potential false-positive measurement. The linear correlation between the two systems is shown in Fig. 8 (right). Deviation from the dotted line indicates poor agreement with the clinical hematology analyzer. Using these metrics, the three time gate plots demonstrated the trend seen previously in Fig. 5 where the deviation between the systems increased between time gates 5 to 6 and 7 to $8 \mathrm{~min}$. As the time gates increased, the lymphocyte count was underestimated while the granulocyte count was overestimated, demonstrated in the Bland-Altman plots (Fig. 8, left). The time gate of 7 to $8 \mathrm{~min}$ had the most deviation, resulting in a bias of 11.30 with a $95 \%$ limits of agreement of -8.29 and 30.89 for the granulocyte population and an $R^{2}$ of 0.758 . Time gates 3 to 4 and 5 to 6 min produced
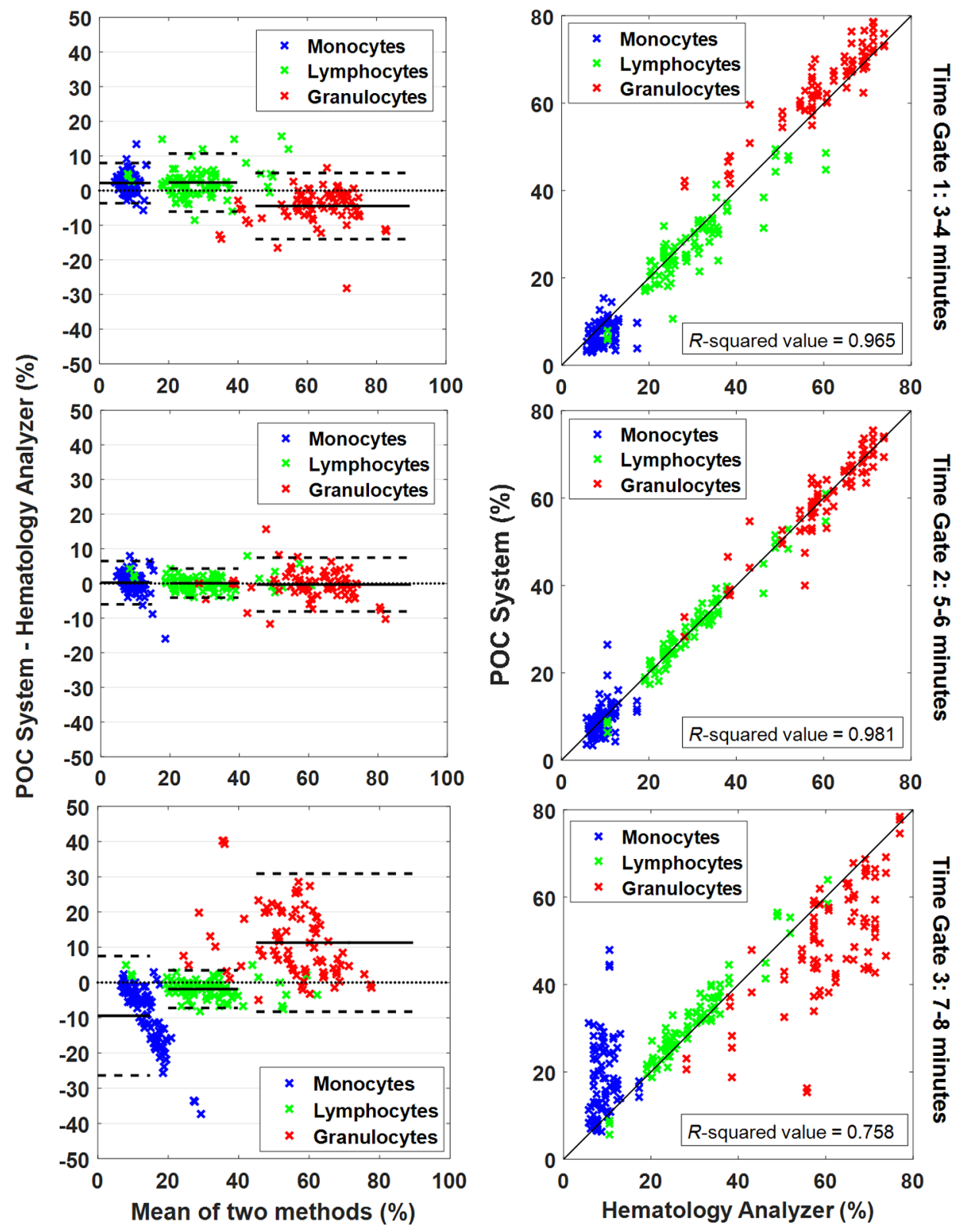

Fig. 8 Bland-Altman analysis and correlation plots for the time gates with an $\mathrm{AO}$ concentration of $10 \mu \mathrm{g} / \mathrm{ml}$ and cutoff values of 0.42 (LM) and 0.58 (MG). Time gate 3 to 4 and 5 to 6 min had similar linear correlation with $R^{2}$ values above 0.960 . The Bland-Altman analysis for time gate 5 to 6 min resulted in the monocytes having a bias of 0.222 and limits of agreement of -6.02 and 6.46 , and lymphocytes and granulocytes producing similar results. Time gate 7 to $8 \mathrm{~min}$ had to greatest inaccuracy with an $R^{2}$ value of 0.750 and the granulocytes with the greatest bias of 11.30 and limits of agreement of -8.29 and 30.89 . 
similar results, yet time gate 5 to 6 min yielded results that were closest to the actual clinical results (bias $=0.222$ and limits of agreement of -6.02 and 6.46 for the monocytes and $\left.R^{2}=0.981\right)$

\section{Discussion}

\subsection{Implications of AO Staining Protocol}

We have demonstrated differences in accuracy when AO concentration and incubation periods (represented by three time gates) are varied. This suggests that a strict staining method is required to produce accurate and consistent results. In this case, using $10 \mu \mathrm{g} / \mathrm{ml} \mathrm{AO}$ and a time gate of 5 to $6 \mathrm{~min}$ with fixed cutoffs at $0.42(\mathrm{LM})$ and $0.58(\mathrm{MG})$ resulted in average deviations of $0.706 \%,-1.534 \%$, and $-0.645 \%$ for lymphocytes, monocytes, and granulocytes, respectively. When the AO concentration and incubation periods were increased a shift in red emission occurred, which correlated to previous publications. ${ }^{33}$ This can be attributed to the accumulation of AO, a cationic dye, in acidic vesicles where the dye becomes "acid-trapped" when it is protonated in a lower $\mathrm{pH}$ environment. ${ }^{36}$ The most important inference from this comparison of multiple staining methods is that a drastic redshift occurs over time that reduces the differential count accuracy and, thus, reduces the effectiveness of the dye in distinguishing the cell populations.

Another potential source of this redshift is due to leukocyte proliferation, in which an increase in DNA/RNA synthesis can be observed in applications using AO to quantify changes in cell cycle phase. ${ }^{34,39}$ For normal leukocytes in peripheral blood, cells are stable in the $G_{0}$ phase and require activation in response to an antigen/mutagen to proceed in the cell cycle. ${ }^{40}$ During the next phases of the cell cycle $\left(\mathrm{G}_{1}\right.$ to $\mathrm{S}$ phase), there is an increase in DNA and RNA syntheses that occurs over the course of many hours $(6+h){ }^{41-43}$ The increase in RNA has been shown to increase the red fluorescence emission intensity of AO-stained leukocytes, whereas the increase in DNA content contributed less to the increase in green fluorescence intensity. ${ }^{43}$ In patients with hematologic malignancies, it may be possible to find some leukocytes in the peripheral blood that exist in a proliferative phase; this may affect the established RG ratio. This could lead to an inaccurate classification, but not necessarily an inaccurate total leukocyte count. Within the environment and time period of the analysis presented in this article, the cells should be in a quiescent state; therefore, the cell cycle should have minimal effect on the red and green fluorescence emissions.

\subsection{Implications of Variations in Postprocessing RG Ratio Cutoff Values}

We used a static approach to separate the cell populations based on the frequency of cells at each RG ratio, where fixed cutoff values were defined by the minimum troughs between the peaks in the histogram and were used for every sample tested; see Fig. 3(d). Our results suggest that, using this approach, slight changes in the cutoffs can change the cell count, which could negatively affect the differential results since we have shown that the peaks can change over time due to the redshift in fluorescence intensities with increased AO concentration (Fig. 6) and incubation periods. An alternative method could potentially involve the use of a dynamic approach where the cutoff values are automatically adjusted based on the shifting peaks that can occur with different staining methods by tracking the troughs or maximum peaks of each cell population for each sample tested. Different factors could affect the definition of the histogram curve, such as cell count and bandwidth of the peaks; therefore, further exploration of that method is necessary.

\subsection{Effect of Time Gating Using an Optimal Differential Method}

Similar to the effects of $\mathrm{AO}$ concentration, different incubation periods, demonstrated by three time gates between 3 and $8 \mathrm{~min}$, can produce different results, as shown in Fig. 8. Our results were comparable to the redshift in $\mathrm{AO}$ emission over time and accumulation of AO previously reported in the literature. ${ }^{33,36}$ Not only was there a redshift within increased incubation periods but the shift did not appear to be uniform (Fig. 6); therefore, controlling accuracy became more difficult when the staining and postprocessing method was not consistently maintained. Also, the Bland-Altman and correlation plots demonstrated that there was a drastic change in test results at the 7 to 8 min time gate; this could be due to a sudden uptake of AO between 6 and 7 min that caused a greater redshift. This suggests that there is a limit to the incubation period where the AO emissions redshift causes all of the cells to become indistinguishable. Additionally, the difference between 3 to 4 and 5 to 6 min time gates were minimal, yet time gate 5 to $6 \mathrm{~min}$ had more test results consistent with the clinical results. Thus, there could still be a difference in results between lower time gates, further demonstrating the need for an optimal staining and postprocessing method.

As current state-of-the-art POC systems rapidly evolve, this information could aid future designs in improving the accuracy and precision of these differential tests. By understanding and quantifying the variations in the red emission, the colorimetric features of AO that are used to distinguish subpopulations of cells for these devices can be utilized more effectively. In general, the method described here is specific to our image-based POC system and AO colorimetric classification; however, this could be extrapolated to other red to green colorimetric POC tests, for instance, image-based methods implementing color cameras and nonimage-based methods such as POC flow cytometry methods implementing microfluidics and fluorescence signal detection using photomultiplier tubes. ${ }^{7,13}$ Additionally, future work could incorporate a five-part differential test to explore whether it can be accurately quantified using AO.

\section{Conclusion}

The information presented in this paper could guide many POC systems using AO as a contrast agent to improve their test accuracy, especially in the monocyte count where most of the inaccuracy lies. We demonstrated that increased concentrations above $10 \mu \mathrm{g} / \mathrm{ml}$ and incubation periods above 6 min negatively affect test accuracy. For the conditions presented here, the time gate 3 to 4 and 5 to $6 \mathrm{~min}$, final $\mathrm{AO}$ concentration $10 \mu \mathrm{g} / \mathrm{ml}$, and RG ratio cutoff values of $0.42(\mathrm{LM})$ and $0.58(\mathrm{MG})$ produced the most accurate and consistent results. Overall, we have quantitatively demonstrated the need for a strict control of the use of AO to stain leukocytes for a three-part differential test to produce results within CLIA standards.

\section{Disclosures}

None of the contributing authors have conflicts of interest, financial or otherwise. 


\section{Acknowledgments}

We would like to thank the Arkansas Biosciences Institute and the Women's Giving Circle for their funding contributions. Also, we thank the staff at the Pat Walker Health Center for their support and participation in this research.

\section{References}

1. C. Briggs, S. Kimber, and L. Green, "Where are we at with point-of-care testing in haematology?" Br. J. Haematol. 158(6), 679-690 (2012).

2. D. Heikali and D. Di Carlo, "A niche for microfluidics in portable hematology analyzers," J. Assoc. Lab. Autom. 15(4), 319-328 (2010).

3. B. Houwen, "The differential cell count," Lab. Hematol. 7, 89-100 (2001).

4. E. K. Amundsen et al., "Absolute neutrophil counts from automated hematology instruments are accurate and precise even at very low levels," Am. J. Clin. Pathol. 137(6), 862-869 (2012).

5. A. O. Gerstner et al., "Comparison of immunophenotyping by slidebased cytometry and by flow cytometry," J. Immunol. Methods 311(1), 130-138 (2006).

6. H. Zhu et al., "Cost-effective and rapid blood analysis on a cell-phone," Lab Chip 13(7), 1282-1288 (2013).

7. C. Majors et al., "Imaging based system for performing a white blood cell count and partial differential at the point of care," in Clinical and Translational Biophotonics, paper TTu2B.3 (2016).

8. S. C. Hur, H. T. K. Tse, and D. Di Carlo, "Sheathless inertial cell ordering for extreme throughput flow cytometry," Lab Chip 10(3), 274-280 (2010).

9. Y. Ben-Yosef et al., "The HemoScreen, a novel haematology analyser for the point of care," J. Clin. Pathol. 69(8), 720-725 (2016).

10. C. van Berkel et al., "Integrated systems for rapid point of care (PoC) blood cell analysis," Lab Chip 11(7), 1249-1255 (2011).

11. H. Zhu et al., "Optofluidic fluorescent imaging cytometry on a cell phone," Anal. Chem. 83(17), 6641-6647 (2011).

12. A. Forcucci et al., "All-plastic miniature fluorescence microscope for point-of-care readout of bead-based bioassays," J. Biomed. Opt. 20(10), 105010 (2015).

13. W. Shi et al., "Four-part leukocyte differential count based on sheathless microflow cytometer and fluorescent dye assay," Lab Chip 13(7), 12571265 (2013).

14. Centers for Disease Control, "Regulations for implementing clinical laboratory improvement amendments of 1988: a summary," J. Am. Med. Assoc. 267(13), 1725-1727 (1992).

15. V. Ng, "Potential waiver of complete blood count/differential testing," CDC, 11 September 2008, https://wwwn.cdc.gov/cliac/pdf/Addenda/ cliac0908/Addendum\%20R.pdf (17 October 2016).

16. R. L. Becker, "Laboratory and clinical issues in CLIA waiver for $\mathrm{CBC} /$ ADCC devices," FDA, 18 July 2008, https://www.fda.gov/ohrms/ dockets/ac/08/slides/2008-4375s1-04\%20-Becker\%2020080718a.pdf (17 October 2016).

17. A. Forcucci et al., "Design and fabrication of miniature objective lens for three part white blood cell differential measurements," Bio-Opt. Des. Appl. BM3A, 5 (2015).

18. A. Banks, Laboratory Tests and Diagnostic Procedures with Nursing Diagnoses, Pearson, Upper Saddle River, New Jersey (2013).

19. P. Allison, "Logistic regression for rare events," Statistical Horizons, 13 February 2012, http://statisticalhorizons.com/logistic-regression-for-rareevents (17 October 2016).

20. C. Majors et al., "Imaging based system for performing a white blood cell count and partial differential at the point of care," Clin. Transl. Biophotonics TTu2B, 3 (2016).

21. Z. J. Smith et al., "Single-step preparation and image-based counting of minute volumes of human blood," Lab Chip 14(16), 3029-3036 (2014).

22. M. R. Hamblin and A. Pinar et al., "Optofluidic lab-on-a-chip devices for photomedicine applications," Chapter 9 in Applications of Nanoscience in Photomedicine, T. Wu et al., Ed., 169-184, Elsevier, Amsterdam, Netherlands (2015).

23. R. Tanabe, S. Hata, and A. Shimokohbe, "MEMS complete blood count sensors designed to reduce noise from electrolysis gas," Microelectron. Eng. 83(4), 1646-1650 (2006).
24. W. Jung et al., "Point-of-care testing (POCT) diagnostic systems using microfluidic lab-on-a-chip technologies," Microelectron. Eng. 132, 46-57 (2015).

25. D. Holmes et al., "Leukocyte analysis and differentiation using high speed microfluidic single cell impedance cytometry," Lab Chip 9(20), 2881-2889 (2009).

26. M. Roy et al., "A simple and low-cost device performing blood cell counting based on lens-free shadow imaging technique," Sens. Actuators B 201, 321-328 (2014).

27. H. V. Pham et al., "Real time blood testing using quantitative phase imaging," PLoS One 8(2), e55676 (2013).

28. O. Mudanyali et al., "Compact, light-weight and cost-effective microscope based on lensless incoherent holography for telemedicine applications," Lab Chip 10(11), 1417-1428 (2010).

29. L. V. Bertalanffy, M. Masin, and F. Masin, "A new and rapid method for diagnosis of vaginal and cervical cancer by fluorescence microscopy," Cancer 11(5), 873-887 (1958).

30. B. A. Lauer, L. B. Reller, and S. Mirrett, "Comparison of acridine orange and Gram stains for detection of microorganisms in cerebrospinal fluid and other clinical specimens," J. Clin. Microbiol. 14(2), 201-205 (1981).

31. S. C. Bhakdi et al., "Re-evaluating acridine orange for rapid flow cytometric enumeration of parasitemia in malaria-infected rodents," Cytometry Part A 71(9), 662-667 (2007).

32. L. V. Bertalanffy et al., "Detection of gynecological cancer; use of fluorescence microscopy to show nucleic acids in malignant growth," Calif. Med. 87(4), 248-251 (1957).

33. F. Traganos and Z. Darzynkiewicz, "Lysosomal proton pump activity: supravital cell staining with acridine orange differentiates leukocyte subpopulations," Methods Cell Biol. 41, 185-194 (1994).

34. F. Traganos et al., "Simultaneous staining of ribonucleic and deoxyribonucleic acids in unfixed cells using acridine orange in a flow cytofluorometric system," J. Histochem. Cytochem. 25(1), 46-56 (1977).

35. S. A. Jahanmehr et al., "Simple technique for fluorescence staining of blood cells with acridine orange," J. Clin. Pathol. 40(8), 926-929 (1987).

36. C. Millot et al., "Characterization of acidic vesicles in multidrug-resistant and sensitive cancer cells by acridine orange staining and confocal microspectrofluorometry," J. Histochem. Cytochem. 45(9), 1255-1264 (1997).

37. C. E. Majors et al., "Low-cost disposable cartridge for performing a white blood cell count and partial differential at the point-of-care," in IEEE Healthcare Innovation Conf. (HIC '14), pp. 10-13 (2014).

38. A. Forcucci et al., "All-plastic, miniature, digital fluorescence microscope for three part white blood cell differential measurements at the point of care," Biomed. Opt. Express 6(11), 4433-4446 (2015).

39. T. Ashihara et al., "A comparison of cell cycle-related changes in postmitotic and quiescent AF8 cells as measured by cytofluorometry after acridine orange staining," Cancer Res. 38(8), 2514-2518 (1978).

40. G. Karp and N. L. Pruitt, "Cellular reproduction," Chapter 14 in Cell and Molecular Biology: Concepts and Experiments, p. 562, Wiley, Hoboken, New Jersey (1999).

41. A. Gothot et al., "Cell cycle-related changes in repopulating capacity of human mobilized peripheral blood CD34(+) cells in non-obese diabetic/ severe combined immune-deficient mice," Blood 92(8), 2641-2649 (1998).

42. Z. Darzynkiewicz et al., "Lymphocyte stimulation: a rapid multiparameter analysis," Proc. Natl. Acad. Sci. U. S. A. 73(8), 2881-2884 (1976).

43. Z. Darzynkiewicz et al., "Relationship between RNA content and progression of lymphocytes through S phase of cell cycle," Proc. Natl. Acad. Sci. U. S. A. 76(1), 358-362 (1979).

Amy J. Powless is a PhD candidate at the University of Arkansas in the Biomedical Engineering Department. She received her BS degree in biological engineering from the University of Arkansas in 2013. She is a trained phlebotomist, which lead to a special interest in blood work and point-of-care applications.

Roxanna J. Conley is a medical laboratory technician at Pat Walker Health Center at the University of Arkansas since 2011. She has been in the field for over 15 years and has experience in hematology, blood bank, urinalysis, coagulation, chemistry, and microbiology. 
Karan A. Freeman is the laboratory manager at Pat Walker Health Center at the University of Arkansas since 1999. She received her BS degree from the University of Arkansas for Medical Sciences in Medical Technology. She has worked as a laboratory technologist and supervisor in quality management and compliance. Also, she has worked as a surveyor for Medicare and Medicaid through CLIA and the Arkansas State Health Department and for the Commission for Accreditation of Rehabilitation Facilities.
Timothy J. Muldoon is an assistant professor of biomedical engineering at the University of Arkansas. He received his BS degree in biomedical engineering from Johns Hopkins University in 2002, his $\mathrm{PhD}$ from Rice University in 2009, and his MD from the Baylor College of Medicine in 2010. His current research interests includes multimodal microendoscopy, multiphoton imaging for gastrointestinal cancer, light sheet confocal microscopy, and whole blood analysis. 